\title{
Optimal design of a bio-inspired anthropocentric shoulder rehabilitator
}

doi:10.1533/abbi.2006.0029

\author{
S. K. Mustafa' ${ }^{1,2}$, G. Yang' ${ }^{2}$, S. H. Yeo ${ }^{1}$ and W. Lin ${ }^{2}$ \\ ${ }^{l}$ School of Mechanical E Aerospace Engineering, Nanyang Technological University, Singapore 639798 \\ ${ }^{2}$ Mechatronics Group, Singapore Institute of Manufacturing Technology, Singapore 638075
}

\begin{abstract}
This paper presents the design of a bio-inspired anthropocentric 7-DOF wearable robotic arm for the purpose of stroke rehabilitation. The proposed arm rehabilitator synergistically utilizes the human arm structure with non-invasive kinematically under-deterministic cable-driven mechanisms to form a completely deterministic structure. It offers the advantages of being lightweight and having high dexterity. Adopting an anthropocentric design concept also allows it to conform to the human anatomical structure. The focus of this paper is on the analysis and design of the 3-DOF-shoulder module, called the shoulder rehabilitator. The design methodology is divided into three main steps: (1) performance evaluation of the cable-driven shoulder rehabilitator, (2) performance requirements of the shoulder joint based on its physiological characteristics and (3) design optimization of the shoulder rehabilitator based on shoulder joint physiological limitations. The aim is to determine a suitable configuration for the development of a shoulder rehabilitator prototype.
\end{abstract}

Key words: Biologically inspired, anthropocentric, arm rehabilitators, cable-driven, parallel mechanism.

\section{INTRODUCTION}

Stroke is a leading cause of severe long-term disability in adults. It is a serious condition that leaves the patient with a high burden of disability and does not have a routinely available restorative treatment. In the last decade, stroke rehabilitation has evolved through an increasing understanding of the neuronal recovery processes. In addition, the introduction of robotic enhancements and virtual reality has further enhanced rehabilitation therapies to carry out intensive and repetitive exercises. In an attempt to enhance the rehabilitation process and supplement the therapists' work, there has been an increasing interest in the use of robotics for rehabilitation (Krebs et al. 1998; Reinkensmeyer et al. 2000; Deutsch et al. 2001; Boian et al. 2002; Fasoli et al. 2003; Kobayashi et al. 2004; Tsagarakis and Caldwell 2003; Kiguchi et al. 2003). These researches are aimed at providing therapists with robotic devices to increase the amount and intensity of

Corresponding Author:

S. K. Mustafa

School of Mechanical \& Aerospace Engineering

Nanyang Technological University

50 Nanyang Avenue, Singapore 639798

Tel: +6567933178

Email: must0003@ntu.edu.sg the movement of the effected limb. This in turn will allow them to focus more on task-specific and complex functional movements.

Statistics has shown that most recovery will happen within the first 6 months after a stroke, as this is the period where rehabilitation is most effective due to spontaneous recovery. Thus physical and occupational therapies are concentrated during this period. Beyond this period, outpatient rehabilitation is given for several more months. Subsequently, little rehabilitation therapy is administered to the stroke patients. In addition, when an arm or leg does not recover function during this early period, most patients compensate for the loss by making negligible attempt to use the impaired limb. This ultimately leads to even greater disability. However, recent studies (Krebs et al. 1998; Reinkensmeyer et al. 2000; Deutsch et al. 2001; Boian et al. 2002; Fasoli et al. 2003) have revealed that such repetitive exercises have been shown to be effective, even in the chronic phase, potentially offering hope to millions of stroke survivors.

One of the most common disabilities in stroke is paralysis of the upper arm. Hence, greater research efforts have been carried out in this area. Some of the well-known robotic arm rehabilitators used in rehabilitation research include the MIT-Manus (Krebs et al. 1998) and the ARM Guide (Reinkensmeyer et al. 2000). The MIT-Manus is a 2-degrees-of-freedom (DOF) robot that assists shoulder and elbow movement by moving the hand and the 


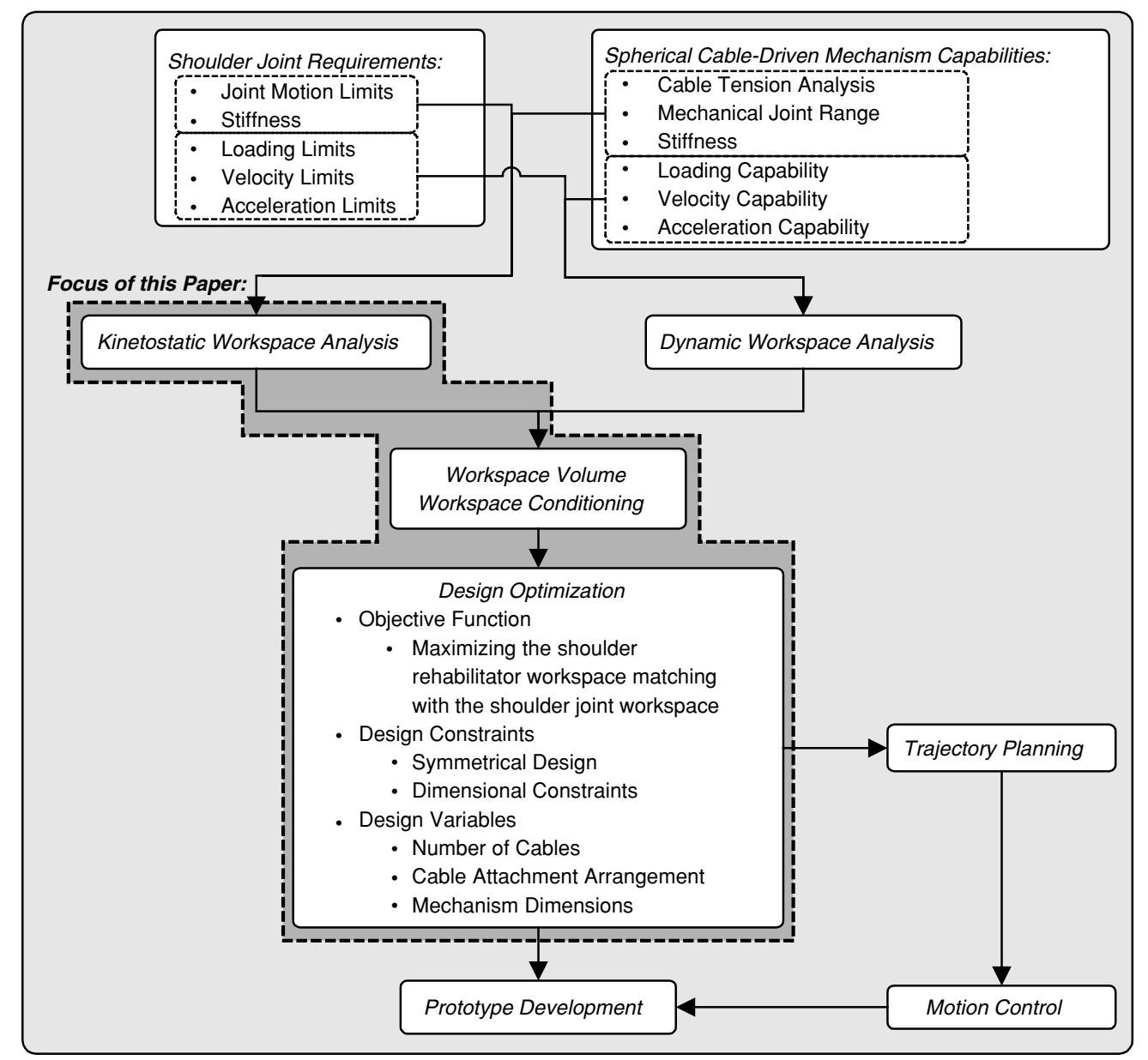

Figure 1 An overview of the development process of the proposed shoulder rehabilitator.

forearm in the horizontal plane. Its unique design feature is its low intrinsic end-point impedance. This allows the device to have free movements as well as active-assist therapy. Similarly, the ARM Guide is a singly actuated 3-DOF device in assisting reaching movements. Its unique feature is that it is statically counter-balanced so that it does not gravitationally load the arm. However, both have limited capabilities as they have a lower DOF as compared to the 7-DOF human arm. Hence, this limits the motion for rehabilitation in certain planes. A novel idea was also proposed by Kobayashi et al. (2004) to develop a muscle suit using McKibben actuators to provide muscular support to the paralyzed. However, it faces numerous challenges in having a limited motion range and issues with slippage and slack of wear. For most of these arm rehabilitators (Krebs et al. 1998; Reinkensmeyer et al. 2000; Tsagarakis and Caldwell 2003; Kiguchi et al. 2003), they were designed with a self-deterministic or adjustable self-deterministic structure. This means that the device generates its own characteristic motion that may not coincide with the characteristic motion of the human structure. In addition, most of them are heavy in weight and fixed at a structure or a wall, which limit the motion of the user's arm. Hence, these devices cannot fully adapt to the user and place additional stresses on their joints while carrying out rehabilitation. This may result in greater discomfort and harm to the user.

In this paper, we focus on the development of a novel 3-DOF bio-inspired anthropocentric shoulder rehabilitator (referred to as shoulder rehabilitator from here onwards), which is part of a novel 7-DOF arm rehabilitator. This paper presents a computationally effective method to analyse equivalent 'force-closure' of cable-driven spherical parallel mechanisms. A shoulder joint workspace representation is also developed based on the task performance requirements (i.e. joint motion limits). This includes a new workspace partition scheme and workspace volume formulation. Global performance evaluation measures utilizing the workspace volume and the stiffness of the mechanism are then proposed for use in design optimization of the shoulder rehabilitator. Figure 1 presents an overview of the development process of the proposed shoulder rehabilitator. At this initial stage, the aim of optimization is to match the cable-driven shoulder rehabilitator capabilities with that of the shoulder joint requirements, with regard to the kinetostatic workspace. Other aspects such as loading, velocity and acceleration limits are not taken into account at this stage. 


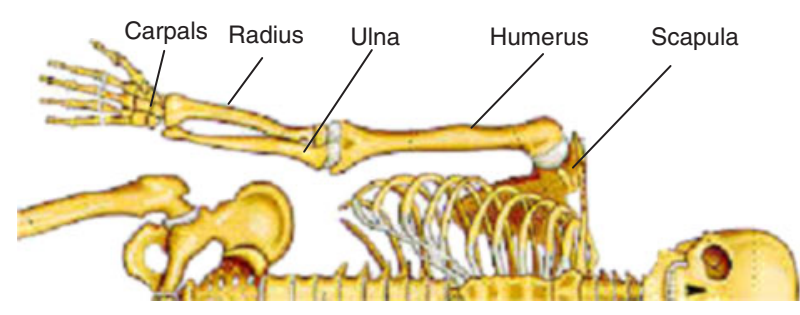

Figure 27-DOF human arm anatomical structure.

The remaining sections of this paper are organized as follows: in the second section, the human arm anatomy is examined for bio-inspired design solutions and the next section presents the conceptual design of the shoulder rehabilitator. Later sections present the cable tension and stiffness analyses. The workspace representation and workspace volume formulation for the human shoulder joint is developed. This section is followed by design optimization of the shoulder rehabilitator, and the paper is summarized in the last section.

\section{HUMAN ARM ANATOMY}

Before coming up with a bio-inspired conceptual design, the human arm anatomy is examined for design solutions. As shown in Figure 2, the human arm is an assemblage of the scapula (shoulder), humerus (upper arm), radius $\&$ ulna (forearm) and carpals (wrist). The arm's major movement is contributed by the relative displacement of these bones. The connections and relative motion of these bones form four basic synovial joints, namely glenohumeral (shoulder), humeroulnar (elbow), radioulnar (forearm) and radiocarpal and midcarpal (wrist) joints. Although the structures of these synovial joints are different, these joints provide similar motions as those in conventional mechanical joints. For kinematic analysis, simplifications have been made to model anatomical joints by using revolute, universal and spherical joints, with fixed centre of rotations since the movements in the human joints' instantaneous centre of rotations are negligible (Lemay and Crago 1996). The shoulder joint is modelled as a spherical joint, where the head of the humerus fits into the concave recess of the scapula (Veeger 2000). The humeroulnar joint is modelled as a revolute joint whose line of rotation is aligned with the centre of the trochlea, while the radioulnar joint is modelled as a cylindrical joint whose axis of rotation passes through the distal end of the ulna and the centre of the capitulum (Lemay and Crago 1996). The wrist joint is modelled as a universal joint because the flexion-extension and the radial-ulna flexion axes are believed to intersect. However, the rotational DOF of the radioulnar joint at the elbow has its axes passing through the distal end of the ulna and the centre of the capitulum to the capitate. Thus, the wrist joint is analogous to a $3-\mathrm{DOF}$ spherical joint while the elbow joint is analogous to a 1-DOF revolute joint. To-

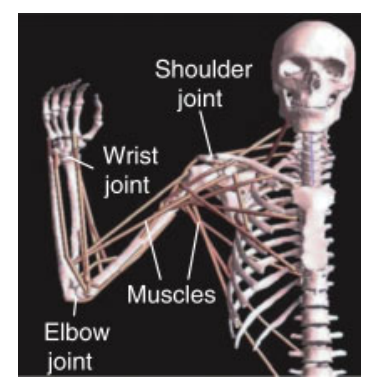

Figure 3 Muscle driving scheme: parallel and redundant.

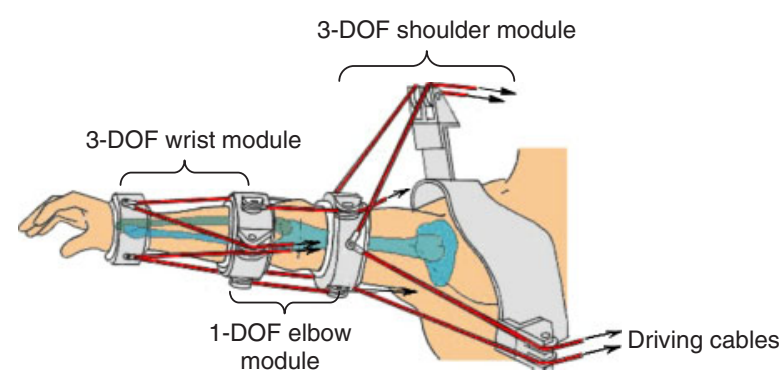

Figure 4 Conceptual design of the biologically inspired anthropocentric cable-driven arm rehabilitator (Yang et al. 2004).

gether with the 3-DOF spherical shoulder joint, the basic human arm forms a 7-DOF structure.

Focusing next on the human arm muscles, the armdriving scheme consists of numerous muscles working in pairs with redundant pairs of muscle to serve as stabilizers, as shown in Figure 3. These pairs of muscles are also arranged in a closed-loop fashion, similar to that of parallel mechanisms. Human muscle physiology is also observed to be of similar characteristics with cables (both having the unilateral property of being only able to pull).

\section{CONCEPTUAL DESIGN}

The conceptual design of a bio-inspired anthropocentric arm rehabilitator is proposed by combining several of these biological solutions mentioned in the previous section (Yang et al. 2004) as shown in Figure 4. The unique feature of the proposed design is the synergistic utilization of the human arm bones and joints with non-invasive kinematically under-deterministic cable-driven mechanical structures. The combined structure forms a complete deterministic mechanism that adapts to the human arm and overcomes the non-compliance issue in existing arm rehabilitators, with improved wearability. In addition, cables are arranged in a fashion similar to the human muscledriving scheme. This makes the proposed arm rehabilitator lighter and compact, as all the actuators are mounted onto the base. It also has greater dexterity due to the presence of redundant cables. From Figure 4, the 3-DOF shoulder module, the 1-DOF elbow module and the 3-DOF wrist module are connected sequentially to form a parallel-inseries 7-DOF arm rehabilitator. 


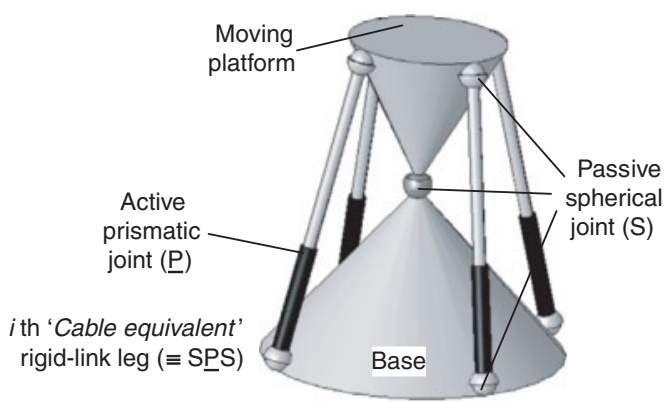

Figure 5 'Cable equivalent' rigid-link kinematic diagram of the shoulder rehabilitator with $m$ cables $(m$ SPS $+1 \mathrm{~S})$.

The human shoulder joint is designed to generate 3DOF spherical motions. Hence, an under-deterministic cable-driven parallel mechanism is designed such that when combined with the shoulder joint, it will produce a deterministic spherical motion, about the human shoulder joint. As shown in Figure 4, the upper arm (moving link) connects with the upper body (fixed base) through the shoulder joint (ball-\&-socket joint) and several driving cables. The cables will 'pull' the upper arm to rotate about the shoulder joint and generate spherical motions.

For rigid-link mechanisms, mobility analysis is carried out using the Gruebler-Kutzback criterion (Tsai 1999, pp. 9-15). However, for cable-driven mechanisms, this criterion can also be utilized to analyse its mobility, provided we convert the cable-driven design to its rigid-link equivalent. Figure 5 shows the equivalent rigid-link kinematic model for the cable-driven shoulder rehabilitator design shown in Figure 4. Based on a modified Gruebler-Kutzback criterion, the mobility of the cable equivalent rigid-link kinematic model of the shoulder rehabilitator is given by

$$
\begin{aligned}
F= & \lambda(n-j-1)+\sum_{i} f_{i}-I_{d} \\
= & 6\{(2 m+2)-(3 m+1)-1\} \\
& +\{m \times(3+3+1)+3\}-m=3
\end{aligned}
$$

where $F$ is the mobility of a mechanism, $\lambda$ is the DOF of the space in which the mechanism is intended to function, $n$ is the number of links in a mechanism, $j$ is the number of joints with $i \mathrm{DOF}, f_{i}$ is the DOF permitted by joint $i, I_{d}$ is the internal passive DOF of the mechanism and $m$ is the number of cables. Note that each end of the 'cable equivalent' rigid-link leg of the proposed shoulder rehabilitator is connected to a ball-\&-socket joint. Hence, a passive rotational DOF exists whose axis passes through both of the ball-\&-socket joints, resulting in one passive DOF for each leg.

It is seen that by converting the cable-driven design to its rigid-link equivalent, the modified Gruebler-Kutzback criterion from Equation (1) is still suitable for mobility analysis of cable-driven mechanisms. In addition, regardless of the number of cables used, the proposed shoulder

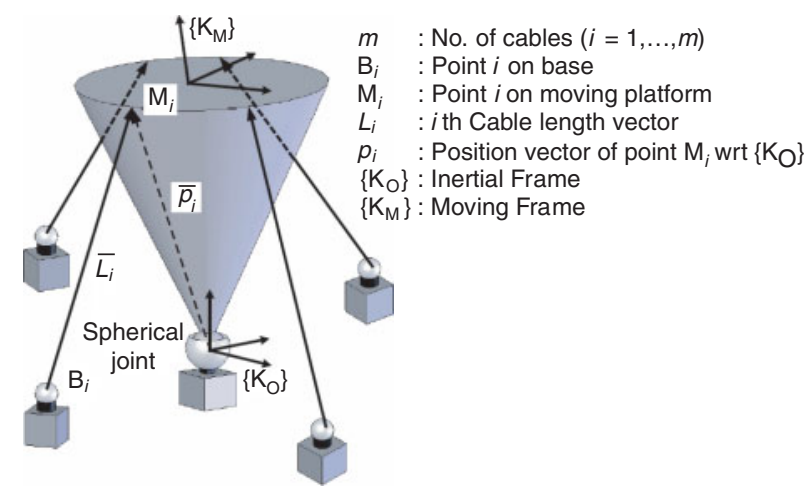

Figure 6 Equivalent kinematic diagram of the shoulder rehabilitator.

rehabilitator has the same mobility (i.e. $3 \mathrm{DOF}$ ) as the 3DOF human shoulder joint.

\section{CABLE-TENSION ANALYSIS}

For a cable-driven mechanism, due to the unilateral driving property of cables, a set of cables must be in positive tension to maintain the end-effector's pose (i.e. force-closed cable tension). Hence, for cable-driven mechanisms, it is vital to carry out cable tension analysis. This is carried out in the following manner.

\section{Determine the cable structure matrix}

From kinetostatic equilibrium analysis, we obtained the following equation of motion for the shoulder rehabilitator at a particular pose:

$$
\mathbf{W}_{\mathrm{Ext}}=A \mathbf{T}
$$

where $\mathbf{W}_{\text {Ext }}$ is the external wrench acting, $\mathbf{T}=$ $\left\{\begin{array}{llll}T_{1} & T_{2} \ldots T_{m}\end{array}\right\}^{T} \in \mathfrak{R}^{m \times 1}$ is the cable tension vector, $\boldsymbol{A}=$ $\left[\begin{array}{llll}a_{1} & a_{2} & \ldots & a_{m}\end{array}\right] \in \mathfrak{R}^{3 \times m}$ is the cable structure matrix, $\boldsymbol{a}_{i}=$ $\left(p_{i} \times \ell_{\mathrm{i}}\right) \in \mathfrak{R}^{3 \times 1}$ is the cable vector and $\ell_{\mathrm{i}}\left(=-\overline{\mathbf{L}}_{i} /\left\|\mathbf{L}_{i}\right\|\right)$ is the unit vector of the $i$ th cable. (From velocity analysis, it can be shown that $A$ is equal to the negative transpose of the Jacobian matrix of rigid-link spherical parallel mechanisms.)

The orientation of frame $\mathbf{K}_{\mathrm{M}}$ on the moving platform, with respect to inertial frame $\mathbf{K}_{\mathrm{O}}$ on the fixed base, is given by the kinematic transformation $T_{\mathrm{O}, \mathrm{M}}=\left[\begin{array}{cc}R & p_{i} \\ 0 & 1\end{array}\right]$, where $\boldsymbol{R}\left(=\boldsymbol{R}_{\mathrm{z}}(\phi) \boldsymbol{R}_{\mathrm{y}}(\theta) \boldsymbol{R}_{\mathrm{z}}(-\phi) \boldsymbol{R}_{\mathrm{Z}^{\prime}}(\sigma)\right)$ is the rotation matrix based on the modified $Z-Y-Z$ Euler angles, i.e. tilt and torsion angles (Bonev and Ryu 1999). In order to withstand any external wrench $\mathbf{W}_{\mathrm{Ext}}$ applied on the moving platform, cables must be able to create tensile forces to achieve kinetostatic equilibrium of the moving platform (see Figure 6). The next step will present a method to evaluate the ability to achieve force-closed cable tension at a particular pose. 


\section{Determine if a set of cables in positive tension exists by evaluating cable structure matrix $A$}

Obtaining the cable structure matrix $A$ from the equation of motion (i.e. Equation (2)), $A$ is evaluated using an equivalent force-closure grasping method derived from the convex analysis. This is because the cable-driven mechanism is very similar to multi-fingered grasping, both of which have a common unilateral driving property (maintaining tension for cables versus maintaining compression for grasping). The equivalent force-closure condition is defined as follows:

The cable tension is force-closure if and only if there does not exist a vector $v \in \mathfrak{R}^{m}, v \neq 0$, such that for $i=1, \ldots$, $4, v^{T} \boldsymbol{a}_{i}=0$ or have the same sign (where $\boldsymbol{a}_{i}$ is $i$ th column vector of cable structure matrix $A$ ).

The cable structure matrix $\boldsymbol{A}$ is evaluated for forceclosed cable tension condition in the following manner:

1. Generate all induced vectors by taking the cross-product of any two different columns such that $a_{i j}=a_{i} \times a_{j}$ $(i, j=1,2, \ldots, m ; i<j)$. In total, ${ }^{m} \mathrm{C}_{2} \boldsymbol{a}_{i j} \mathrm{~s}$ are generated.

2. Take dot products of $\boldsymbol{a}_{i j}$ with $\boldsymbol{a}_{k}(k=1,2, \ldots, m ; k \neq$ $i ; k \neq j)$, a column vector $\boldsymbol{b}_{i j}$ with $(m-2)$ elements is formed. In total, ${ }^{m} \mathrm{C}_{2} b_{i j}$ s are formed.

3. Check each elements of $\boldsymbol{b}_{i j}$. If the elements for any $\boldsymbol{b}_{i j}$ have different signs (i.e. at least contain one positive and one negative element), this means that the force-closed cable tension condition is satisfied. Else, it is not satisfied.

This approach results in a simple and fast computation method to determine force-closed cable tension for the shoulder rehabilitator with $m$ cables. Note that if the cables can achieve force-closure (i.e. a set of positive cable tension) at a particular pose, it means that the mechanism does not suffer from any singularities at this pose. Hence, singularity analysis is not required.

\section{STIFFNESS ANALYSIS}

In addition to cables having unilateral driving properties, they are also flexible. It is therefore vital to maintain sufficient stiffness at each pose in order to achieve the desired positional accuracy. For cable-driven mechanisms, stiffness analysis is carried out in the following manner:

\section{Determine the stiffness matrix}

Applying the principle of virtual work under kinetostatic equilibrium (Tsai 1999), the stiffness matrix $S$ at a particular pose is given as

$$
S=k^{\prime} A L_{0}^{-1} A^{T}
$$

where $k^{\prime}$ is the stiffness per reciprocal unit length and $L_{0}^{-1}=\operatorname{diag}\left(L_{01}^{-1}, \ldots L_{0 m}^{-1}\right)$ is a diagonal matrix whose elements are the reciprocal of the original cable lengths. Given the pose of the moving platform, $L_{0}^{-1}$ can be calculated from the actual cable length (Verhoeven et al. 1998)

$$
\begin{aligned}
\boldsymbol{L}_{0 i}^{-1} & =L_{@ \text { Tension } T_{i}}^{-1}\left(1+\left(k^{\prime}\right)^{-1} T_{i}\right) \\
& (i=1,2, \ldots, m) .
\end{aligned}
$$

(In practice, the $\left(k^{\prime}\right)^{-1} T_{i}$ terms are normally less than $0.5 \%$ and are ignored.)

\section{Determine the stiffness condition index of the stiffness matrix $S$}

As shown by Strang (1976), the condition number of a matrix is used in numerical analysis to estimate the error generated in the solution of a linear system of equations by the error on the data. When applied to the Jacobian matrix, the condition number gives a measure of the accuracy of the Cartesian velocity and the static load acting on the end of the end-effectors. Similarly, the condition number of the stiffness matrix $S$ can also be used to give a measure of accuracy for cable-driven mechanisms, which includes the effect of cable lengths. The stiffness condition index, i.e. SCI (which is the reciprocal of the condition number), is given as the ratio of the minimum to the maximum singular value of $S$ at a given pose. SCI ranges from 0 to 1. Large values for SCI (close to 1) ensure good stiffness with respect to force and velocity transmission, while SCI values close to 0 indicate poor stiffness.

\section{WORKSPACE ANALYSIS}

The workspace of any mechanism is a pertinent issue in the context of optimum kinematic design. This is especially critical in the case of cable-driven parallel mechanisms due to the unilateral property and the flexible nature of cables. These introduce additional constraints which further limit its workspace. The manoeuvrable workspace is defined as a set of poses that satisfy the following three conditions simultaneously:

1. The forward/inverse displacement solutions exist.

2. A set of cables must be in positive tension to maintain the end-effector's pose (i.e. force-closed cable tension).

3. It must be within the human arm joint limits.

For the shoulder rehabilitator workspace analysis, the critical issues are the representation of the orientation workspace, with regard to force-closed cable tension and the shoulder joint motion limits.

\section{Workspace representation}

Discretization algorithms are typically utilized to numerically analyse the mechanism's workspace which consists of discretizing the three-dimensional space, solving the inverse kinematics at each pose and verifying the constraints that limit the workspace. Such discretization algorithms are popular in majority of researches and can be applied to any type of architecture. In biomechanics, however, workspace representation for the purpose of defining the range of motion is approached differently as compared to 


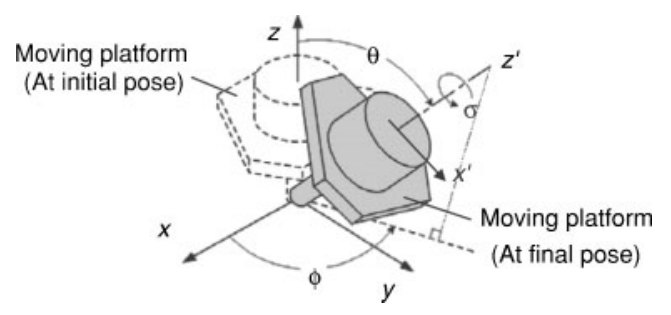

Figure 7 Tilt and torsion angles description (adapted from Bonev and Ryu (1999)).

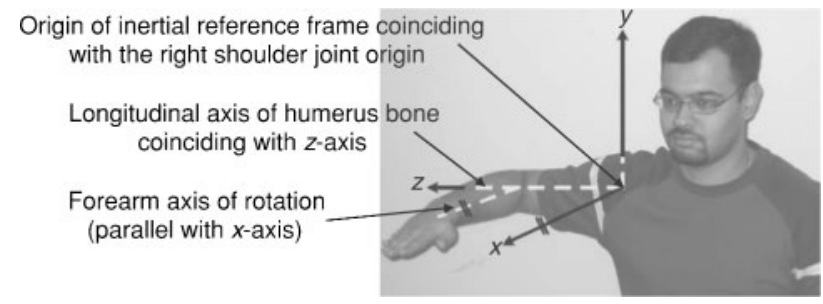

Figure 8 Initial pose of the right upper arm at $\sigma=0$.

mechanisms or computer animations. While quaternion seems to be used as the standard method for representing motion in computer animations, they are not so often used in biomechanics. Quaternion has the advantages in terms of lack of gimbal lock and insensitivity to round-off errors, but they suffer from problems of interpretation in terms of meaningfully clinical or anatomical angles. Euler angles, on the other hand, allow some degree of intuition for the rotation angles, but suffer from singularities. Representations using equivalent angle-axis have also been proposed (Korein 1984; Cheng et al. 2000), but the main issue for biomechanics is that interpretation of joint motions must have an intuitive feel for clinical studies while being mathematically tractable at the same time. Hence, there is a great debate as to the use of Euler angles with quaternion and equivalent angle-axis for workspace representations (Baerlocher 2001).

In this paper, we utilize an existing orientation representation approach (known as the tilt and torsion angles) proposed by Bonev and Ryu (1999) to represent the workspace of the human shoulder joint based on its joint range of motion limits. The advantage of this representation is that it is able to separate the 3-DOF orientations into two components: the 2-DOF tilt and the 1-DOF twist. The tilt component $(\phi$ and $\theta$ ) determines the direction of the upper arm, while the twist component $(\sigma)$ determines the rotation about itself, as shown in Figure 7. This representation is very intuitive for clinical studies, and the joint motion limit boundaries can be easily identified using this representation.

The origin of the inertial frame coordinate system is at the shoulder joint (as shown in Figure 8), and the orthogonal axes $x, y$ and $z$ are described as

- $x$ : pointing to the front direction of the body,

- $y$ : pointing to the upward direction of the body and

- $z$ : cross-product of $x$ with $y$.

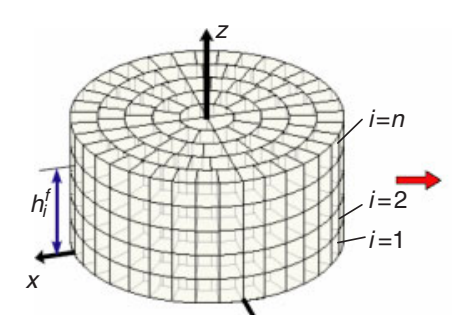

(a)

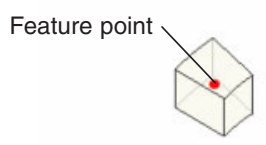

(d)

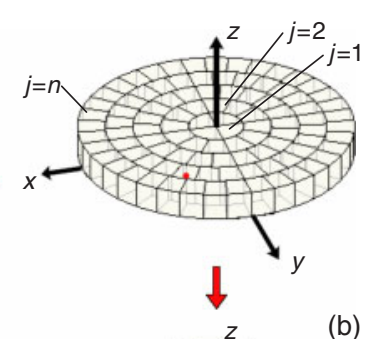

(b)
Figure 9 (a) 3D view of cylindrical workspace, (b) division scheme of $i$ th circular disc, (c) division scheme of $j$ th circular band in $i$ th disc, and (d) an element in the cylindrical workspace.

As for the moving frame coordinate system, the origin is at the shoulder joint and the orthogonal axes $x^{\prime}, y^{\prime}$ and $z^{\prime}$ are described as

- $x^{\prime}$ : pointing along the predefined forearm axis of rotation and based on the initial pose of the upper arm (as shown in Figure 8),

- $y^{\prime}$ : cross-product of $z^{\prime}$ with $x^{\prime}$ and

- $z^{\prime}$ : pointing along the longitudinal axis of the humerus bone at any pose.

The angles $\phi, \theta$ and $\sigma$ are described as

- $\phi$ : angle between the base $x$-axis and the projection of the approach vector onto the base $x y$-plane,

- $\theta$ : angle between base $z$-axis and the moving $z^{\prime}$-axis and

- $\sigma$ : angle of rotation of the upper arm about the moving $z^{\prime}$-axis with respect to the predefined $x^{\prime}$-axis reference line.

Gosselin and Wang (2004) proposed an intuitive way to display orientation workspace using a cylindrical coordinate system. Adopting a similar approach, the orientation workspace of the shoulder rehabilitator is displayed using a system of cylindrical coordinates $\{r, \gamma, h\} . \phi, \theta$ and $\sigma$ will be the polar angle $\gamma$, the polar radius $r$ and the height $h$, respectively. It has a 'one-to-one' mapping between the points in the cylindrical plot and the poses in the task space. The equi-volumetric partition scheme of the cylindrical workspace of radius $\pi$ and height $\pi$ (with $\phi \in\left[-180^{\circ}, 180^{\circ}\right], \theta \in\left[0^{\circ}, 180^{\circ}\right]$ and $\left.\sigma \in\left[0^{\circ}, 180^{\circ}\right]\right)$ is attained as follows:

Step 1: As shown in Figure 9(a), the solid cylinder is divided along $y$-axis into $n$ identical circular discs of height $\delta h=\pi / n$. Starting from the bottom, the discs are then labelled from 1 to $n$, i.e. $i=1, \ldots, n$. This results in $n$ circular discs.

Step 2: As shown in Figure 9(b), each circular disc $i$ is then divided into $n$ circular bands of thickness $\delta r=\pi / n$ along the radial direction as shown in Figure 9(b). Note that the first circular band is actually a circular disc of radius $\pi / n$ and height $\pi / n$. Starting from this 


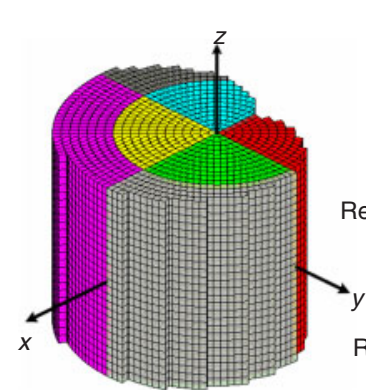

(a)

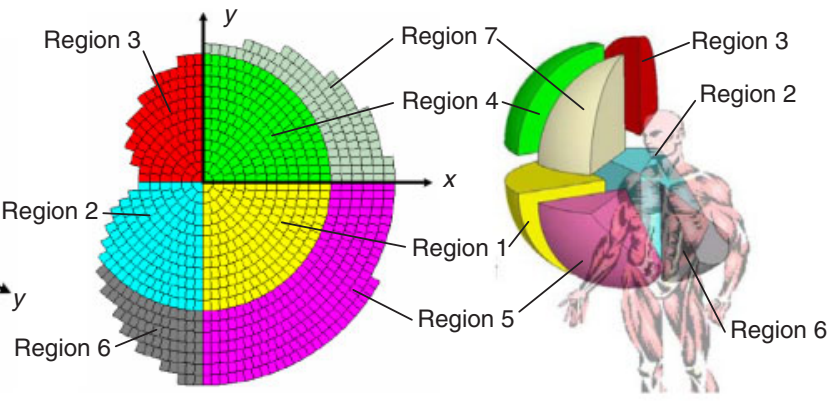

(b)

(c)

Figure 10 (a) 3D view of the right shoulder joint workspace (with $n=25$ ), (b) cross-sectional view of $i$ th disc of the right shoulder joint workspace, and (c) the corresponding regions in the Cartesian task space at $\sigma=0$. The colours indicate the regions in the cylindrical workspace plot to the corresponding regions in the Cartesian task space.

central circular disc, the circular bands are labelled from 1 to $n$, i.e. $j=1, \ldots, n$. This results in $n$ circular bands.

Step 3: As shown in Figure 9(c), the central circular disc (i.e. $j=1$ ) is divided into four quadrants, where each quadrant is regarded as the basic element of volume $\delta V_{\text {Cartesian }}=\pi^{4} / 4 n^{3}$. For each circular band $j$ (i.e. $j=2, \ldots, n$ ), it is circumferentially divided into $4(2 j-1)$ equi-volumetric element of volume $\delta V_{\text {Cartesian }}$ with same spacing angle of $\pi / 2(2 j-1)$, as shown in Figure 9(d). Starting from the $x$-axis, the elements are labelled counter-clockwise from 1 to $4(2 j-1)$, i.e. $k=1, \ldots, 4(2 j-1)$. This results in $\sum_{j=1}^{n} 4(2 j-1)=4 n^{2}$ elements for each circular disc.

In total, there are $4 n^{3}$ elements in the cylindrical workspace and each element is specified by its feature point $f_{i j k}$ using a triplet index $(i, j, k) . f_{i j k}$ is selected as the intersection point of the three neutral planes of the element and is given as

$$
f_{i j k}=\left[x_{i j k}, y_{i j k}, z_{i j k}\right]=\left[r_{i j}^{f} \cos \gamma_{i j k}^{f}, r_{i j}^{f} \sin \gamma_{i j k}^{f}, h_{i}^{f}\right]
$$

where $\quad h_{i}^{f}=(2 i-1) \pi / 2 n, \quad r_{i j}^{f}=(2 j-1) \pi / 2 n \quad$ and $\gamma_{i j k}^{f}=(2 k-1) \pi / 4(2 j-1)($ for $i=1, \ldots, n ; j=1, \ldots, n$; and $k=1, \ldots, 4(2 j-1))$. When the number of elements is sufficiently large, each element can be locally represented by $f_{i j k}$. The advantage of this division scheme is that the singularity points for the tilt and torsion angles at $\theta=0$ and $\pi$ are avoided. In addition, all elements are geometrically identical, with simplified analysis and computational effort, and even the workspace for the shoulder joint can easily be determined, given the joint motion limits.

Based on the shoulder joint motion range (Hamilton and Luttgens 2002), the approximated maximum range of the tilt angles and twist angles is $\phi \in\left[-180^{\circ}, 180^{\circ}\right], \theta \in$ $\left[0^{\circ}, 140^{\circ}\right]$ and $\sigma \in\left[-90^{\circ}, 90^{\circ}\right]$, respectively. These values are approximated as they vary slightly between each individual. By limiting the workspace of the shoulder module to the shoulder joint motion limits, its boundary is a cylinder of height $\pi$ (i.e. from $-\pi / 2$ to $\pi / 2$ ) with an irregular shaped cross-section. Figure 10 shows the threedimensional (3D) view and the irregular shaped crosssection of the right shoulder joint workspace, as well as the corresponding region in the Cartesian task space.

\section{Workspace volume}

In robotics, the special orthogonal group $\mathrm{SO}(3)$ is commonly used to describe rigid-body rotations. $\mathrm{SO}(3)$ denotes a group of $3 \times 3$ real orthogonal matrices with unit determinant. When evaluating the workspace volume, due to nonlinear parameterization, the volume of $\mathrm{SO}(3)$ (i.e. $V_{\mathrm{SO} 3}$ ) is not equal to the geometrical volume of the cylindrical workspace (i.e. $\left.V_{\text {Cartesian }}\right)$. Hence, a volume-associated integral factor $\left(I_{v}\right)$ has to be introduced to accurately determine $V_{\mathrm{SO} 3}$ from $V_{\text {Cartesian }}$. From the concept of metrics, $I_{v}$ is defined as the absolute value of the Jacobian matrix which relates the rate of change of the orientation parameters to describe the rotation to the angular velocity of the rigid body. When computing the volume of $\mathrm{SO}(3)$ from the cylindrical coordinate representation of tilt and torsion angles, an equivalent $I_{\nu}$ is determined as $\sin \theta / \theta$ by adopting a similar approach presented by Chirikjian and Kyatkin (2001). The volume for the discretized $\mathrm{SO}(3)$ workspace is now given as

$$
V_{\mathrm{SO}(3)}=\left(\sum_{i=1}^{h_{\text {total }}} I_{\nu i}\right) \Delta V_{\text {Cartesian }}=\left(\sum_{i=1}^{h_{\text {total }}} \frac{\sin \theta_{i}}{\theta_{i}}\right)\left(\frac{\pi^{4}}{4 n^{3}}\right)
$$

where $h_{\text {total }}$ is the total number of poses (i.e. feature point) and $\theta_{i}$ is the $\theta$ angle at the $i$ th feature point, with respect to the cylindrical shoulder joint workspace shown in Figure 10.

\section{DESIGN OPTIMIZATION}

In such anthropocentric mechanisms, the critical issue is the matching of the mechanism characteristics with the human body characteristics. For this reason, the first step in achieving an optimally designed mechanism is to match 
the workspace of the mechanism closest possible with that of the workspace generated by the human joints, with a minimum amount of stiffness. This section will present two global workspace evaluation indices, one utilizing the workspace volume and the other utilizing mechanism stiffness. This will be followed by the design optimization of the shoulder rehabilitator.

\section{Workspace evaluation index}

Two global workspace evaluation indices are presented, namely the workspace-matching index and the global stiffness conditioning index. The former is a quantitative evaluation index, while the latter is a qualitative evaluation index.

\section{Workspace matching index}

For the shoulder rehabilitator, the main objective for design optimization is to achieve a workspace as close as possible to the actual shoulder joint workspace. Hence, this performance evaluation criterion utilizes the workspace volume. This index, i.e. workspace matching index (WMI), is given as

\section{$W M I$}

$=\frac{\text { Manoeuverable workspace volume of the mechanism }}{\text { Total workspace volume of the shoulder joint }}$

$=\frac{\sum_{i=1}^{n_{a}} \frac{\sin \theta_{i}}{\theta_{i}}}{\sum_{j=1}^{n_{\text {total }}} \frac{\sin \theta_{j}}{\theta_{j}}}$

where $n_{a}$ is the number of poses achievable by the mechanism within the shoulder joint workspace while $n_{\text {total }}$ is the total number of poses, with respect to the shoulder joint workspace. WMI ranges from 0 to 1 . A WMI value 1 (ideal) indicates that the shoulder rehabilitator is able to achieve all the poses in the shoulder joint workspace. However, the shoulder rehabilitator can have attainable poses that are beyond the workspace defined by the shoulder joint motion limits. These poses are of no importance and should be avoided as the human shoulder joint is unable to achieve these poses in the first place.

\section{Global stiffness conditioning index}

For the shoulder rehabilitator, it utilizes cables which are flexible. Hence, stiffness is greatly affected and it is very critical to maintain sufficient stiffness. For the design optimization, stiffness will be utilized as a secondary criterion. Adopting a similar approach as the global conditioning index proposed by Yang et al. (2003), the global stiffness conditioning index (GSCI) is proposed as a performance quality measure for the cable-driven shoulder rehabilitator. GSCI is the integration of SCI over the whole workspace and is given numerically by

$\mathrm{GSCI}=\frac{\sum_{q=1}^{n_{a}} \operatorname{SCI}(S)_{\mathrm{q}} \Delta V_{\mathrm{SO} 3}}{\sum_{q=1}^{n_{a}} \Delta V_{\mathrm{SO} 3}}=\frac{\sum_{q=1}^{n_{a}}\left(\mathrm{SCI}(S)_{\mathrm{q}} \frac{\sin \theta_{i}}{\theta_{i}}\right)}{\sum_{q=1}^{n_{a}} \frac{\sin \theta_{i}}{\theta_{i}}}$.
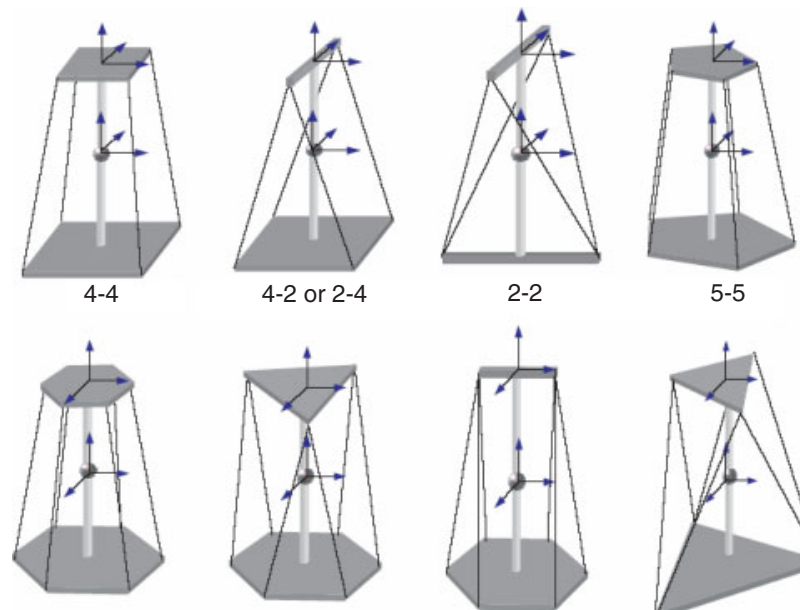

6-6

6-3 or 3-6
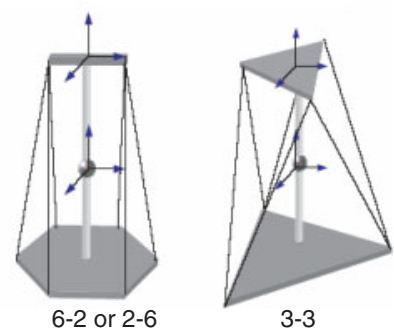

Figure 11 Configurations of the shoulder rehabilitator with 4, 5 and 6 cables.

GSCI ranges from 0 to 1 . Large values for GSCI (close to 1) ensure good overall stiffness with respect to force and velocity transmission, while GSCI values close to 0 indicate poor overall stiffness.

\section{Optimized configuration}

In any mechanism design process, the idea of optimality is challenging to realize, as there is no single global criterion that will include all the objective functions. It is practically impossible to include workspace quantity and quality measures together as a single performance measure. For the shoulder rehabilitator, the objective is to achieve maximization of the WMI (i.e. to match the rehabilitator workspace as close as possible to the shoulder joint workspace). However, it is also desired to have the best conditioning (i.e. SCI) of the workspace, but these two goals are often conflicting. Hence, GSCI will be used as a secondary criterion to assist in selecting the best between mechanisms with the same WMI.

Design variables include the number of cables, the cable attachment arrangement and the mechanism dimensions (i.e. $d_{1}, d_{2}, r_{1}$ and $r_{2}$, which are the length of the base and moving platform constraint links, and the radius of the base and moving platform, respectively). However, these design variables will result in an infinite number of configurations to investigate. Hence, with an aim of finding a realizable solution, the optimization process is subjected to the following design constraints:

1. Symmetric design (i.e. with regular polygonal shapes for the cable attachment points on the base and moving platform) with the same number of cables in each attachment point group. The suitable range for the minimum number of cables needed to manipulate a spherical cable-driven mechanism is determined based on Caratheodory and Steinitz theorems (Murray et al. 1994). For a $n$-DOF parallel cable-driven mechanism, Caratheodory's theorem implies that a minimum of $(n+$ 1) cables in positive tension are required, while Steinitz's 


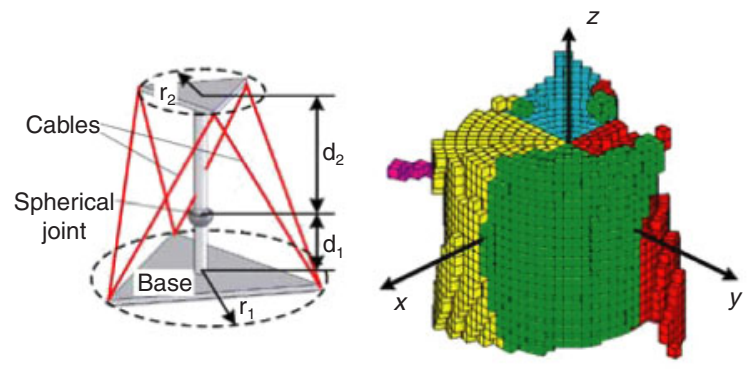

(a)

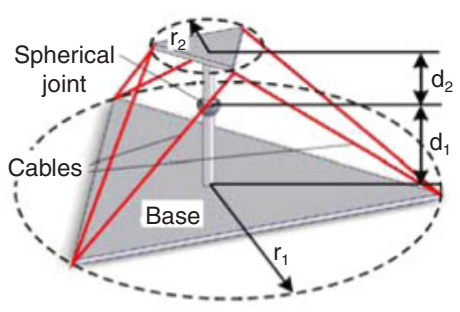

(b)

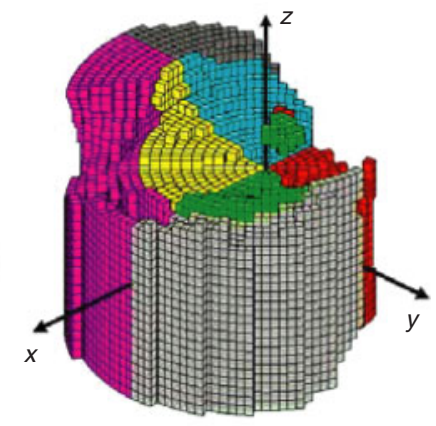

Figure 12 (a) Initial configuration of the shoulder rehabilitator with $r_{1}=200 \mathrm{~mm}, r_{2}=100 \mathrm{~mm}, d_{1}=100 \mathrm{~mm}, d_{2}=200 \mathrm{~mm}$ and its cylindrical workspace plot $(\mathrm{WMI}=0.361$ and $\mathrm{GSCI}=0.111)$. (b) Optimized configuration of the shoulder rehabilitator with $r_{1}=398 \mathrm{~mm}, r_{2}=103 \mathrm{~mm}, d_{1}=148 \mathrm{~mm}, d_{2}=96 \mathrm{~mm}$ and its optimized cylindrical workspace plot $(\mathrm{WMI}=0.707$ and $\mathrm{GSCI}=0.294)$.

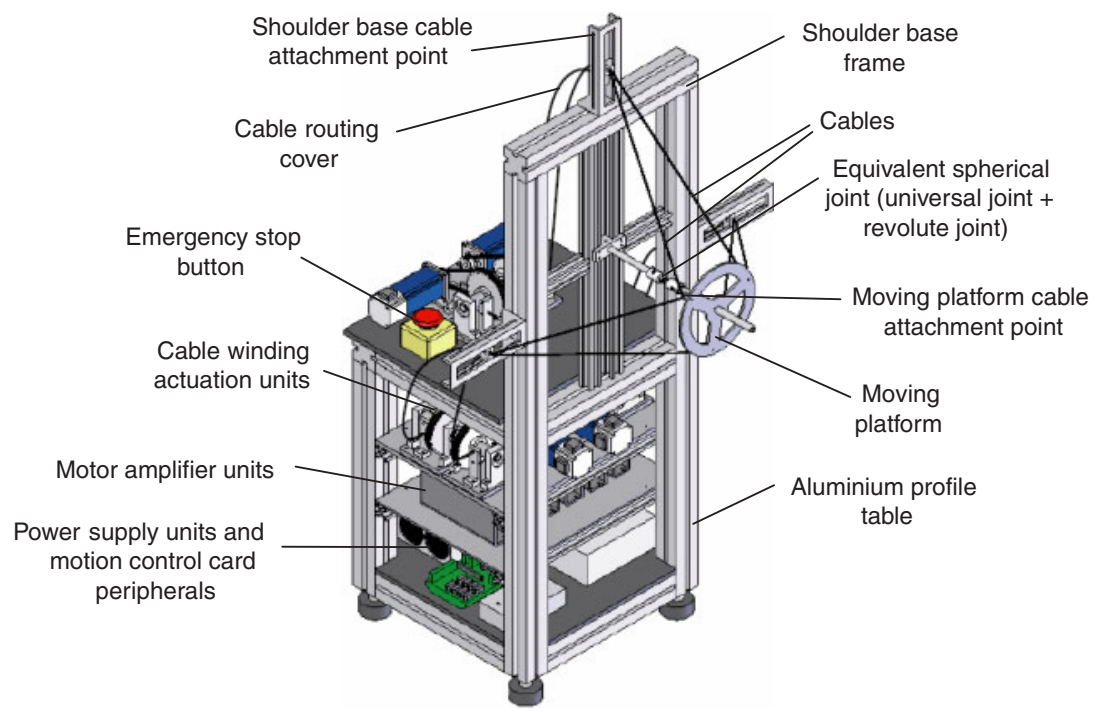

Figure 13 CAD of the proof-of-concept shoulder rehabilitator prototype based on the optimization results obtained.

theorem implies that at most $2 n$ cables in positive tension are required. Hence, the minimum number of cables needed to effectively manipulate the shoulder rehabilitator is from four to six. Figure 11 shows the various configurations of the shoulder rehabilitator with 4,5 and 6 cables.

2. $200 \mathrm{~mm} \leq r_{1} \leq 400 \mathrm{~mm}, 100 \mathrm{~mm} \leq r_{2} \leq 150 \mathrm{~mm}$, $0 \mathrm{~mm} \leq \bar{d}_{1} \leq 200 \mathrm{~mm}$ and $50 \mathrm{~mm} \leq d_{2} \leq 300 \mathrm{~mm}$. These dimensional constraints are based on the human arm anthropometric data. They ensure that the shoulder rehabilitator will not be too large or too small for practical application.

Due to the non-linearity of the objective function, a modified complex search method (Deb 1998) is utilized for the optimization of the various shoulder rehabilitator configurations shown in Figure 11. After optimization, the most suitable configuration for the shoulder rehabilitator is $3-3$, with WMI of 0.707 , GSCI of 0.294 , and optimized dimensions of $r_{1}=398 \mathrm{~mm}, r_{2}=103 \mathrm{~mm}, d_{1}=148 \mathrm{~mm}$ and $d_{2}=$ $96 \mathrm{~mm}$. Figure 12 shows the initial and the optimized 3-3 shoulder rehabilitator configurations and their respective cylindrical workspace plots. Figure 13 shows the CAD of the proof-of-concept shoulder rehabilitator prototype based on the optimization results. This is currently being fabricated.

\section{CONCLUSION}

In this paper, the main focus is to present a novel design methodology for a 3-DOF shoulder rehabilitator based on the task performance requirements. Drawing inspirations from bio-inspired solutions, an anthropocentric design was proposed. This design combines an under-deterministic cable-driven parallel mechanism with the human shoulder joint to form a complete deterministic structure. The design methodology was divided into three main steps. The first step was to analyse the kinetostatic workspace of the cable-driven shoulder rehabilitator. A computationally effective cable-tension analysis algorithm was proposed in view of the duality of the cable-tension with multi-fingered grasping. Due to the flexible nature of cables, stiffness analysis was carried out and a mechanism stiffness evaluation 
method based on the conditioning index of the stiffness matrix was also proposed.

The next step was to determine the task performance requirements i.e. the shoulder joint workspace based on its joint motion limits. Being able to intuitively describe the pose of the upper arm based on the shoulder joint angles, the tilt and torsion angle was adapted to represent the poses in the shoulder joint workspace. This workspace was then discretized using a simple yet effective partition scheme and quantified to obtain some critical performance measures for the shoulder rehabilitator design.

The final step was to carry out design optimization to determine the optimal configuration for the shoulder rehabilitator, such that the shoulder rehabilitator workspace will closely match the shoulder joint workspace. Two global performance evaluation indices were proposed. The first is the workspace matching index, which utilized the workspace volume, and the second is the global stiffness conditioning index, which utilized the stiffness matrix of the mechanism. Optimization was carried out using these two global performance evaluation indices, which resulted in a 3-3 six-cable configuration with its optimized dimensions. Ongoing works include the proof-of-concept prototype development of the shoulder rehabilitator (shown in Figure 13). Future work will focus on the control system algorithm formulation and implementation. This is a part of a large research project to ultimately develop a 7-DOF arm rehabilitator for stroke rehabilitation.

\section{ACKNOWLEDGMENTS}

Mustafa S. K. would like to thank the Agency of Science, Technology \& Research (A*STAR) for awarding the PhD scholarship, and the technical support provided by Singapore Institute of Manufacturing Technology of A*STAR and Robotics Research Centre of NTU. The authors would also like to thank Prof. Chen I-Ming from the School of MAE, NTU and Mrs Susan Niam from the Physiotherapy Department, Tan Tock Seng Hospital, Singapore.

\section{REFERENCES}

Baerlocher P. 2001. Inverse Kinematics Techniques for the Interactive Posture Control of Articulated Figures. Lausanne: Ecole Polytechnique Federale De Lausanne.

Boian RF, Lee CS, Deutsch JE. et al. 2002. Virtual reality-based system for ankle rehabilitation post stroke. In 1st International Workshop on Virtual Reality Rehabilitation (Mental Health, Neurological, Physical, Vocational), Lausanne, Switzerland.

Bonev IA, Ryu J. 1999. Orientation workspace analysis of 6-DOF parallel manipulators. In ASME Design Engineering Technical Conferences, Las Vegas, NV.

Cheng PL, Nicol AC, Paul JP. 2000. Determination of axial rotation angles of limb segments-A new method. 7 Biomech, 33: 837-43.

Chirikjian GS, Kyatkin AB. 2001. Engineering applications of noncommutative harmonic analysis. Boca Raton, FL: CRC Press.
Deb K. 1998. Optimization for Engineering Design: Algorithms and Examples. New Delhi: Prentice-Hall.

Deutsch JE, Latonio J, Burdea GC. et al. 2001. Post-stroke rehabilitation with the Rytgers ankle system: A case study. Presence (MIT), 10:416-30.

Fasoli SE, Krebs HI, Stein J. et al. 2003. Effects of robotic therapy on motor impairment and recovery in chronic stroke. Arch Phys Med Rehabil, 84:477-82.

Gosselin CM, Wang J. 2004. Kinematic analysis and design of cable-driven spherical parallel mechanisms. In Proceedings of the 15th CISM-IFToMM Symposium on Robot Design, Dynamics and Control, Montreal, Canada.

Hamilton N, Luttgens K. 2002. Kinesiology: Scientific Basis of Human Motion, 10th ed. New York: Mc-Graw Hill.

Kiguchi K, Tanaka T, Watanabe K. et al. 2003. Design and control of an exoskeleton system for human upper-limb motion assist. In IEEE/ASME International Conference on Advanced Intelligent Mechatronics.

Kobayashi H, Uchimura A, Shiiba T. 2004. Development of muscle suit for upper body. In IEEE International Conference on Robotics \& Automation, New Orleans, LA.

Korein JU. 1984. A Geometrical Investigation of Reach. ACM distinguished dissertation, Cambridge, MA: The MIT Press.

Krebs HI, Hogan N, Aisen ML. et al. 1998. Robot-aided neurorehabilitation. IEEE Trans Rehabil Eng, 6(1): 75-87.

Lemay MA, Crago PE. 1996. A dynamic model for simulating movements of the elbow, forearm and wrist. 7 Biomech, 29 : 1319-30.

Ming A, Higuchi T. 1994. Study of multiple degree-of-freedom positioning mech using wires, Part 1-Concept, design and control. Int 7 Japan Soc Prec Eng, 28:131-8.

Murray RM, Li Z, Sastry SS. 1994. A Mathematical Introduction to Robotic Manipulation. Boca Raton, FL: CRC Press.

Reinkensmeyer DJ, Kahn LE, Averbuch M. et al. 2000. Understanding and treating arm movement impairment after chronic brain injury: Progress with the arm guide. 7 Rehabil Res Dev, 37(6): 653-62.

Rockafellar RT. 1970. Convex Analysis. Princeton, NJ: Princeton University Press.

Strang G. 1976. Linear Algebra and its Applications. New York: Academic Press.

Tsagarakis NG, Caldwell DG. 2003. Development and control of a 'soft-actuated' exoskeleton for use in physiotherapy and training. Auton Robots, 15: 21-33.

Tsai L-W. 1999. Robotic analysis: The mechanics of serial and parallel manipulators. New York: Wiley.

Veeger HEJ. 2000. The position of the rotation center of the glenohumeral joint. 7 Biomech, 33: 1711-5.

Verhoeven R, Hiller M, Tadokoro S. 1998. Workspace, stiffness, singularities and classification of tendon-driven Stewart platforms. In 6th International Symposium on Advances in Robot Kinematics, Strobl, Austria.

Yang G, Ho HL, Lin W. et al. 2003. A differential geometry approach for workspace analysis of spherical parallel manipulators. In 11th World Congress in Mechanism and Machine Science, Tianjin, People's Republic of China.

Yang G, Ho HL, Chen W. et al. 2004. A haptic device wearable on a human arm. In Proceedings of 2004 IEEE Conference on Robotics, Automation And Mechatronics, Singapore, pp. 243-8. 

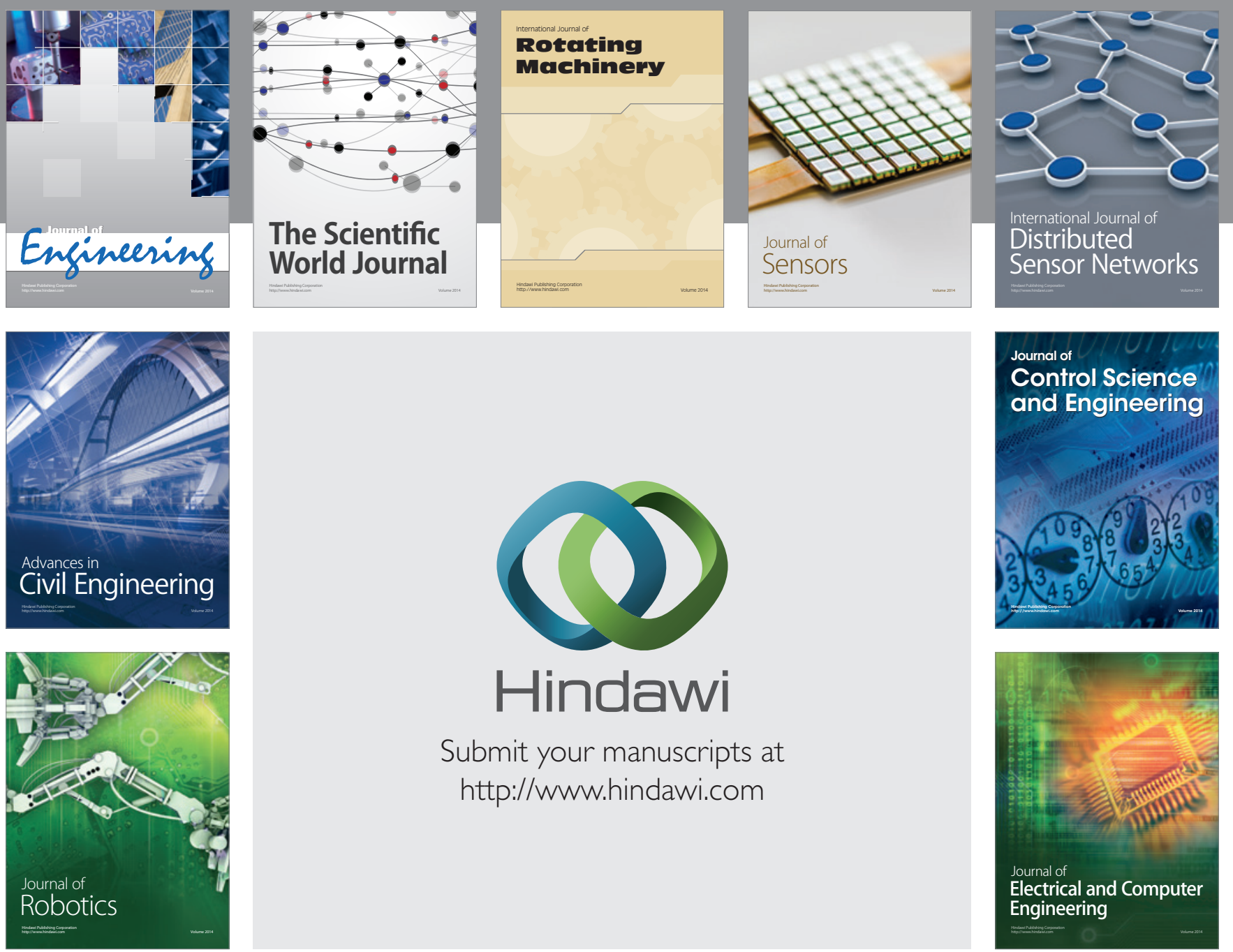

Submit your manuscripts at

http://www.hindawi.com
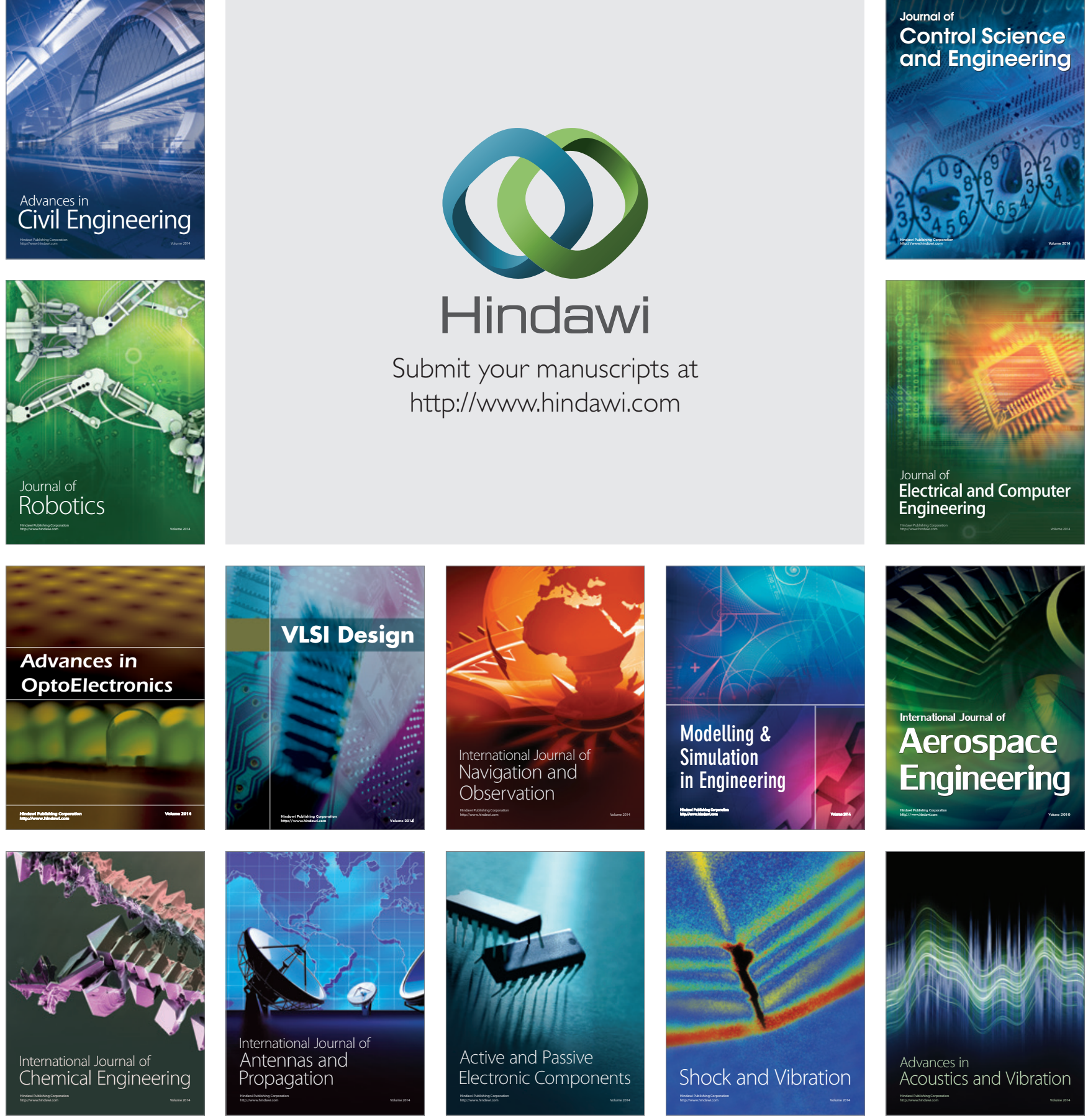